\title{
Targeting TRPV3 for the Development of Novel Analgesics
}

\author{
Susan M. Huang ${ }^{1}$ and Man-Kyo Chung*,2 \\ ${ }^{I}$ Neuroscience Research, Global Pharmaceutical Research and Development, Abbott Laboratories, Abbott Park, IL \\ 60064, USA; ${ }^{2}$ Department of Neural and Pain Sciences, School of Dentistry, Program in Neuroscience, University of \\ Maryland, 650 W. Baltimore Street, Baltimore, MD 21201, USA
}

\begin{abstract}
Decades of characterization of the transient receptor potential vanilloid subtype 1 (TRPV1) have led to the realization of its central role in thermosensation and pain perception. A large number of pharmaceutical companies have had interest in developing TPRV1 antagonists for the treatment of pain. The subsequent discovery of multiple other members of this TRPV family has not gone unnoticed. TRPV3 exhibits approximately $40 \%$ homology to TRPV1, and has common as well as distinct features from TRPV1 in channel physiology, expression and function. Here we review the current understanding of TRPV 3 channel biology, activation, sensitization and the consequences of TRPV 3 manipulation for thermosensation and nociception, as well as additional considerations regarding the expression of TRPV 3 in the skin. We weigh in on the available evidence in the context of potential development of TRPV3 modulating agents as analgesics.
\end{abstract}

Keywords: TRPV3, Novel Analgesics, thermosensation, pain perception.

\section{INTRODUCTION}

Transient receptor potential vanilloid subtype 3 (TRPV3) is a member of the TRP vanilloid subtype family. TRPV3 was first discovered in humans and mice by three research groups working independently [1-3]. The amino acid sequence of TRPV3 shows significant homology with TRPV1, the capsaicin receptor, by approximately $40 \%$ in humans $[1,2]$. Like TRPV1, TRPV3 is predicted to have six transmembrane domains, a pore loop between fifth and sixth transmembrane domains, and three ankyrin repeats in the amino terminal domain [2]. Despite moderate homogy of 40\%, TRPV3 and TRPV1 show distinct tissue expression, electrophysiological and pharmacological properties, which suggest potentially distinct contributions to nociception in vivo.

Previously identified siblings, TRPV1 and TRPV2, are activated by noxious temperatures $[4,5]$. Since these two receptors are primarily localized in sensory ganglia, they were hypothesized to be sensors of painful heat in sensory neurons. TRPV3 was the first receptor identified in the TRP channel family that is activated by warm temperatures. In contrast to TRPV1 and TPRV2, it is primarily localized in skin keratinocytes. The expression of TRPV3 in keratinocytes was confirmed in both humans and rodents at the transcript, protein and functional levels [3, 6-13]. Besides keratinocytes, TRPV3 was also detected in other epithelia in tongue, palate, nose, hair follicle, and distal colon in humans or rodents $[9,14,15]$. However, the expression of TRPV3 protein in sensory ganglia is reported only in humans and

*Address correspondence to this author at the Department of Neural and Pain Sciences, School of Dentistry, Program in Neuroscience, University of Maryland, 650 W. Baltimore Street, Baltimore, MD 21201, USA;

Tel: 410-706-4452; Fax: 410-706-0865; E-mail: mchung@umaryland.edu monkeys $[1,2,6]$, but not in rodents where only evidence at the transcript level exists. These reports point to some important considerations for studying the role of TRPV 3 in thermosensation and pain. First, the sensory role of TRPV3 may be indirect and involve interactions between epithelial cells and sensory neurons as previously hypothesized by several groups of investigators $[3,16]$. Second, the mechanisms and the extent of contribution of TRPV 3 to thermosensation and nociception may not be identical in rodents and humans. Therefore the translation of results from rodents to humans may not be straightforward.

\section{Activation of TRPV3}

\section{General Characteristics of TRPV3 Currents}

TRPV3 is a non selective cationic channel and its activation leads to the permeation of monovalent and divalent cations through the channel pore. $\mathrm{Ca}^{2+}$ permeates TRPV3 at a higher rate than $\mathrm{Na}^{+}$and the relative permeability of $\mathrm{Ca}^{2+}$ to $\mathrm{Na}^{+}$is approximately $10-12[2,17]$. The current-voltage relationship shows strong outward rectification such that current amplitudes over negative potential ranges are much smaller than those over positive potentials. Like TRPV1, activation of TRPV 3 also shows voltage-dependence and opening probability increased at depolarized potentials [17-19]. Chemical or thermal agonistic stimuli shift the voltage dependence to the left so that gating occurs more frequently even at lower voltages [17-20]. Single channel conductance of TRPV3 is 150 to 200 pS $[2,17]$.

\section{Heat}

TRPV3 was first identified as a receptor that can be activated by warm temperatures. Heterologously expressed recombinant TRPV3 starts to be activated at 31 to $39^{\circ} \mathrm{C}$ with $Q_{10}$ (increase in reaction rate per $10^{\circ} \mathrm{C}$ increase in 
temperature) of 6.6 to 17.3 [1-3]. Similar warm temperature activation of TRPV3 was also demonstrated in cultured keratinocytes [10]. In contrast to these findings, a recent study reports high heat threshold of TRPV3 $\left(>50{ }^{\circ} \mathrm{C}\right)[21]$. Although the source of the discrepancy is unclear, different rate, duration or extent of heating might have affected the extent of activation of TRPV3.

\section{Chemical Agonist}

TRPV3 is reported to be activated by multiple different chemical agonists. 2-Aminoethyl diphenylborinate (2-APB) and its structural analog diphenylboronic anhydride (DPBA) activate TRPV3 efficaciously $[17,22,23]$. But these compounds also activate other TRP channels including TRPV1 and TRPV2 [17, 22]. Natural plant-derived monoterpene compounds activate TRPV3. Camphor, carvacrol, menthol, eugenol, carveol, thymol were demonstrated to activate TRPV3 in heterologous systems and in native cells, such as tongue epithelial cells or keratinocytes $[15,24-26]$. These compounds are not selective to TRPV3 and activate other members of TRP channels including TRPV1, TRPM8 and TRPA1 as well [15, 25, 27, 28]. Citral can similarly activate TRPV3, TRPV1, TRPM8 and TRPA1 [29]. Recently, incensole acetate was reported to show strong activation of TRPV3 with modest effects in TRPV1 and TRPV4 [30]. Plant cannabinoids, such as $\Delta(9)$-tetrahydrocannabinol and cannabidiol, have also been reported to activate TRPV3 [31]. An endogenous molecule, farnesyl pyrophosphate (FPP), which is an intermediate product of a mavelonate pathway for cholesterol synthesis has been reported to activate TRPV3 but not other TRPV channels [32].

\section{Structural Basis of Activation}

Although the structural mechanisms underlying TRPV3 activation are not clearly understood, recent studies provided important information. Unbiased screening of random mutants of TRPV3 revealed that the pore region of TRPV3 is critical for heat activation [19]. Mutations of five amino acid residues within this pore domain and the sixth transmembrane domain specifically ablate heat activation of TRPV3, with little effects on chemical or voltage activation. A similar approach revealed that two residues in amino and carboxy terminal domains, H426 and R696, are specifically involved in the activation of TRPV3 by 2-APB [33]. These two residues are not conserved in TRPV4 which is not activated by 2 -APB. Mutation of the two variant residues of TRPV4 to the two residues of TRPV3 confers sensitivity to 2-APB in TRPV4. These studies strongly suggest that chemical and thermal activation of TRPV3 likely have differential structural bases. Further analysis of functional structures would facilitate the development of therapeutics targeting agonist- or pathology-specific activation of TRPV3.

\section{Potential Endogenous Modulators}

Since mammalian TRPV3 can be activated by temperatures close to body temperature, it is important to identify factors enhancing or attenuating the activity of TRPV3 under pathophysiological conditions. Like many other thermosensitive TRP channels, simultaneous application of heat and the chemical agonists described above strongly potentiates the TRPV3 activity [15, 23, 24]. Besides exogenous agonists, the activity of TRPV3 can also be modulated by multiple factors that are regulated during inflammation or tissue injury. Many inflammatory mediators released at the site of injury can sensitize TRPV1 through $G$ protein-coupled receptor (GPCR)-mediated phospholipase $\mathrm{C}$ (PLC)-dependent mechanisms, which underlie thermal hyperalgesia [34]. TRPV3 can also be sensitized by multiple agonists of GPCRs, such as ATP, histamine, bradykinin, and acetylcholine, when TRPV3 is heterologously expressed with their corresponding receptors. Receptor-mediated sensitization involves the depletion of the membrane lipid phosphatidylinositol $[4,5]$ bisphosphate $\left(\mathrm{PI}(4,5) \mathrm{P}_{2}\right)[18]$. The depletion of PI $(4,5) \mathrm{P}_{2}$ results in enhanced agonist activation of TRPV3 that is evidenced by a decrease in thermal threshold, a leftward shift in concentrationdependent relationship for 2-APB and an alteration in voltage-dependence. Such tonic inhibition by PI $(4,5) \mathrm{P}_{2}$, which can be relieved by the PLC- $\beta$-mediated breakdown of PI $(4,5) \mathrm{P}_{2}$, is reminiscent of the receptor-mediated sensitization of TRPV1 [35]. TRPV3 function can also be enhanced by the stimulation of receptor tyrosine kinase by epidermal growth factor (EGF) or transforming growth factor- $\beta$ (TGF- $\beta$ ) Activation of epidermal growth factor receptor (EGFR) enhances agonist-induced activation of keratinocyte TRPV3 in PLC- $\gamma$ - dependent manner [8]. Since PLC- $\gamma$ also hydrolyze PI $(4,5) \mathrm{P}_{2}$, it is possible that the sensitization of TRPV3 may also be through similar mechanisms by PLC- $\beta$.

In contrast to the inhibitory effects of $\mathrm{PI}(4,5) \mathrm{P}_{2}$ on TRPV3, unsaturated fatty acid such as arachidonic acid, potentiates the agonist-induced response of TRPV3 [36]. The potentiating effects are not due to the metabolism of arachidonic acid [36], which is different from the effects on TRPV1 which can be attributed to metabolites such as 12HPETE, rather than arachidonic acid itself [37]. These lipidmediated mechanisms could potentially modulate the function of TRPV3 under inflammatory conditions and generate pathophysiological signals in keratinocytes.

Mechanisms of TRPV1 sensitization through phosphorylation have been widely studied. Sensitization resulting from inflammatory mediators acting on their perspective GPCRs involves the action of protein kinases to phosphorylate various amino acid residues of TRPV1[34]. Agonist activation of TRPV3 could be enhanced by treatment with an activator of protein kinase $\mathrm{C}(\mathrm{PKC})$, phorbol myristate acetate [36]. It is not known if such effects are the consequences of phosphorylation of TRPV3, and further investigation is required.

\section{Sensitizing Properties of TRPV3}

A unique property defining the activation of TRPV3 is its sensitization characteristics. The amplitude of channel current progressively increases as heat stimuli are repeated or prolonged $[1-3,10]$. This phenomenon is in sharp contrasts to other heat-gated TRP channels such as TRPV1, TRPA1 or TRPV4, which shows robust desensitization by repeated or prolonged stimuli $[10,16,38]$. Later, it was demonstrated that chemical agonists also sensitize TRPV3 in a similar manner to heat $[15,22-24]$. The sensitizing activity 


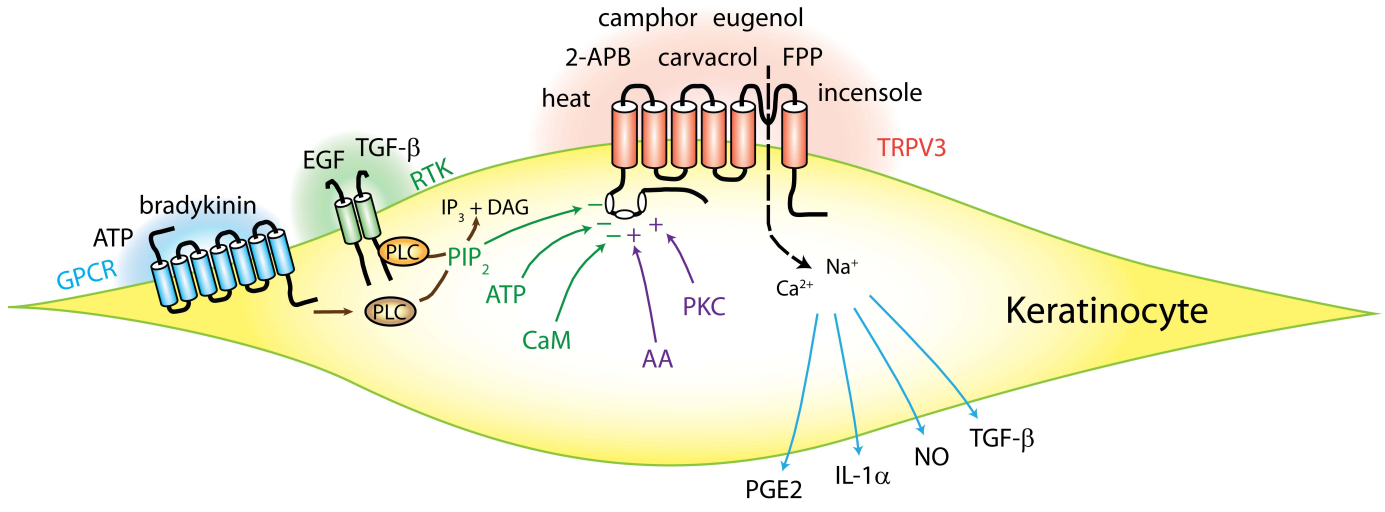

Fig. (1). Schematics of activation, regulation and functions of transient receptor potential vanilloid subtype 3 (TRPV3) in keratinocytes. Chemical and thermal agonists activate TRPV3 in keratinocytes. G-protein coupled receptors (GPCR), receptor tyrosine kinase (RTK), and other intracellular factors modulate the functions of TRPV3. TRPV3 activation induces the release of potential signaling molecules such as prostaglandins and cytokines. See text for references. 2-APB, 2-aminoethyl diphenylborinate; AA, arachidonic acid; ATP, adenosine triphosphate; CaM, calmodulin; EGF, epidermal growth factor; FPP, farnesyl pyrophosphate; IL-1 $\alpha$, interleukin $1 \alpha$; NO nitric oxide; PGE 2 , prostaglandin $\mathrm{E}_{2} ; \mathrm{PIP}_{2}$, phosphatidylinositol $(4,5)$ bisphosphate; PKC, protein kinase C; PLC, phospholipase C; TGF- $\beta$, transforming growth factor- $\beta$.

of TRPV3 by repeated stimuli is important since it may contribute to the generation of progressively larger excitatory signals in response to the repeated stimuli, leading to a higher level of activity within the nociceptive system in the continued presence of thermal or other noxious stimuli. The underlying mechanisms of sensitization appear to be complicated. The roles for extracellular and intracellular $\mathrm{Ca}^{2+}$ were proposed. In this model, TRPV3 is tonically inhibited by both extracellular and intracellular $\mathrm{Ca}^{2+}$ through distinct mechanisms. Extracellular $\mathrm{Ca}^{2+}$ inhibits TRPV3 through D641 which is located at the outer mouth of channel pore $[17,20]$. Calmodulin binds to the middle of amino terminal domain of TRPV3 (amino acid 108-130) and intracellular $\mathrm{Ca}^{2+}$ inhibits TRPV3 through calmodulin [20]. As agonist stimulation continues, such $\mathrm{Ca}^{2+}$-dependent inhibitions may be progressively attenuated and the activity of TRPV3 progressively enhanced. Interaction of calmodulin with amino terminal domain of TRPV3 was confirmed by another study [39]. Calmodulin mainly interact with the ankyrin repeat domain located in the amino terminal domain at K169 and K174. These sites are 'multiligand binding sites', and intracellular ATP also binds to the same residues [39]. Such multiligand binding sites are conserved in TRPV1 [40]. In TRPV1, $\mathrm{Ca}^{2+} /$ calmodulin desensitize the channel while ATP enhances function. However, since both ATP and calmodulin suppress the activity of TRPV3, the functional effects of ATP and calmodulin on TRPV3 appear to be more complicated [20,39].

Sensitization of TRPV3 also occurs in the absence of $\mathrm{Ca}^{2+}$, suggesting $\mathrm{Ca}^{2+}$-independent mechanisms $[17,41]$. $\mathrm{Ca}^{2+}$-independent sensitization is thought to be related to the hysteresis of TRPV3 gating induced by irreversible structural changes during activation [41]. In this model, the rate of activation of TRPV3 is very slow and does not reach steady state even during minutes of application of the agonist. Therefore repeated or prolonged application of agonist induces accumulated activation of TRPV3 that results in sensitization. It will be important to investigate the mechanisms of TRPV3 activation involving both reversible and irreversible events.

In addition to the mechanisms outlined, when TRPV3 is subjected to prolonged or excessive agonist stimulation it can enter a different mode of activation. An Initial gradually sensitizing phase $\left(\mathrm{I}_{1}\right)$ is frequently followed by an abrupt transition to a secondary phase $\left(\mathrm{I}_{2}\right)$ [17]. Such transition was reported following the application of thermal or chemical agonists $[17,19,39]$. The secondary phase is characterized by the loss of outward rectification, altered voltage and temperature dependence, and decrease in ruthenium red sensitivity [17]. The transition from $I_{1}$ to $I_{2}$ phase is irreversible and apparently associated with changes in the TRPV3 pore region, since ionic selectivity of TRPV3 is altered during $\mathrm{I}_{2}$ phase and mutation of D641 in the pore domain facilitates the transition [17]. Decreased inhibition by $\mathrm{Ca}^{2+}$ at the pore accompanies such transition. Although the structural basis related to such biphasic activity of TRPV3 is not known, it is worthwhile to note that TRPV1 and TRPA1 also display alteration in ionic selectivity upon agonist stimulation, suggesting changes in the pore regions of these channels $[42,43]$. We speculate that pore regions of these channels, including TRPV3, may participate as a gate during activation with differing extent of contribution and time course. Further investigation is needed to clarify the structural mechanisms of TRPV3 sensitization and biphasic activation.

\section{Cellular Consequences of TRPV3 Activation}

Intercellular signaling mechanisms between nonneuronal cells and sensory nerves is a well-known mode of sensory transduction in other sensory modalities such as taste 
[44] and hearing [45, 46]. Since TRPV3 is mainly expressed in keratinocytes, intercellular signaling mechanisms between keratinocytes and sensory nerve terminals have been considered, and cellular consequences following the activation of TRPV3 in keratinocytes examined. It was demonstrated that chemical or thermal TRPV3 agonists in keratinocytes induce release of potential signaling molecules such as prostaglandin $\mathrm{E}_{2}\left(\mathrm{PGE}_{2}\right)$, adenosine triphosphate (ATP), nitric oxide (NO), interleukin $1 \alpha$ (IL- $1 \alpha$, TGF- $\beta$ [8, $12,15,47]$. Although the details of the mechanisms leading to release of these molecules following TRPV3 activation is not entirely clear, it can be postulated that TRPV3 stimulation leads to $\mathrm{Ca}^{2+}$-dependent signaling such as exocytosis or activation of enzymes. For example, chemical and thermal agonists of TRPV3 induce $\mathrm{Ca}^{2+}$-dependent activation of cyclooxygenase and release of prostaglandin $E_{2}$ from keratinocytes [12]. Upregulation of TGF- $\beta$ and transglutaminases in keratinocytes are also well-known $\mathrm{Ca}^{2+}$ dependent processes that depend on TRPV3 [8]. Unlike canonical NO synthesis via NO synthase (NOS), TRPV3dependent NO synthesis in keratinocytes is neither dependent on $\mathrm{Ca}^{2+}$ nor NOS, but on intracellular acidification and nitrite [47]. These molecules released from keratinocytes are presumed to affect nearby keratinocytes or sensory nerve endings projected to the skin. ATP released from keratinocytes by TRPV3 agonists was demonstrated to activate sensory neurons in in vitro co-culture preparation. The contributions of these molecules in an in vivo setting have been addressed to some extent and are reviewed below, although the details still await elucidation. Activation, regulation and functions of TRPV3 in keratinocytes are summarized in Fig. (1).

\section{Inhibition of TRPV3}

Limited published data exists on inhibitors of TRPV3. Ruthenium red and 2,2-diphenyltetrahydrofuran (DPTHF), a structural analog of 2-APB, suppress TRPV3, but neither are specific for TRPV3 [17]. Isopentenyl pyrophosphate (IPP), another metabolite of the mevalonate pathway, suppresses TRPV3 as well as TRPA1 [48]. Resolvin D1 and resolvin E1 display potent antinociceptive and antihyperalgesic effects $[49,50]$. One of the potential anti-nociceptive mechanisms of the resolvins is to suppress TRP channels. 17S-resolvin D1 was found to inhibit TRPV3 as well as TRPV1 and TRPA1 [51]. However, a stereoisomer 17R-resolvin D1 shows specific suppression of TRPV3 [52].

\section{Involvement of TRPV3 in Thermosensation and Nociception \\ Rationale for a Role of TRPV3 in Thermosensation and Nociception}

Interest in potential contributions of TRPV3 to thermosensation and nociception likely stemmed from TRPV3's ability to be activated by warm temperatures when expressed heterologously [1-3], its tissue expression pattern that included keratinocytes, as well as its high degree of homology to TRPV1 $[1,2]$, which is well recognized for a role in thermosensation and nociception $[4,53]$. It seemed plausible that a channel capable of responding to temperature changes and localized at the interface with the environment could be involved in warm temperature perception and nociception.

\section{Keratinocyte TPRV3 Involvement in Thermosensory Transduction}

Although TRPV3 immunoreactivity was described in human dorsal root ganglia (DRG) [1], and TRPV3 mRNA detected in peripheral neuronal tissues $[1,2]$, no functional TRPV3 responses have been described from rodent DRG neurons. Unlike TRPV1 which is heavily expressed on small diameter sensory neurons $[4,38]$, TRPV 3 is more prominently expressed in skin keratinocytes [1-3], and TRPV3mediated currents and calcium influx have been recorded from keratinocytes $[10,11]$. This arrangement led to the hypothesis that TRPV3 in keratinocytes might participate in perception via a relay of information to sensory nerve endings through chemical mediators $[3,54,55]$. Several groups have attempted to answer this question with various approaches.

With mice overexpressing TRPV3 in keratinocytes, Huang and colleagues [12] showed that activation of TRPV3 in keratinocytes can result in the release of $\mathrm{PGE}_{2}$, a small molecule well known for its role in sensitizing nerve endings. When TRPV1 contribution was masked, overexpression of keratinocyte TRPV3 led to greater thermal hyperalgesia that was abolished by inhibition of prostaglandin synthesis, indicating that keratinocyte TRPV3 can facilitate thermal nociception by the release of $\mathrm{PGE}_{2}$. It remains to be investigated if this occurs under normal physiological levels of TRPV3 expression. Independently, Mandadi and colleagues [56] found that heat could induce ATP release from keratinocytes, and ATP levels were reduced in TRPV3 deficient cells. The released ATP could activate DRG neurons in a co-culture arrangement. Although no behavioral data were reported, the results suggest keratinocyte TRPV3-mediated ATP release could potentially act on peripheral nerves and modulate sensory perception. A subsequent report by Miyamoto and colleagues [47] found that another consequence of heat activation of TRPV3 is NO production. Suppression of NO synthesis abolished the hyperalgesia found in female wildtype and TRPV3 deficient 129S1/SVImJ ice. The data indicate TRPV3-stimulated NO production likely contributes to thermosensory behavior in certain genetic backgrounds. Hence, it appears that stimulation of keratinocyte TRPV3 could result in $\mathrm{PGE}_{2}$ or ATP release, or NO production, and some or all of which may have a role in modulating nociception under certain circumstances.

\section{Effects of Genetic Ablation of TPRV3 on Thermal Sensation and Nociception}

Owing to the lack of selective and efficacious TRPV3 agonists or antagonists, genetically modified animals has served an indispensible role in advancing our understanding of the physiological roles of TRPV3, particularly in the in vivo setting. Moqrich and colleagues [24] showed TRPV3 deficient mice were impaired in tests of innocuous temperature perception and acute thermal nociception. The mice assayed were on a mixed genetic background and consisted of females and males. They subsequently isolated the effect to females in the 129S1/SVImJ background, but not in males nor in the C57BL6 background [47]. In agreement with the Moqrich study [24], male mice deficient for both TRPV3 and TRPV4 on the C57BL6 background 
had minimal impairment in both innocuous temperature perception and thermal nociception [57]. Therefore, data from these genetic ablation studies indicate that there is likely limited contribution of endogenous TRPV3 in innocuous temperature perception and acute thermal nociception, and its role appears to be dependent upon genetic background and gender.

\section{Effects of TRPV3 Agonists and Antagonists on Nociception}

Although compounds identified as TRPV3 agonists and antagonists exist, there is not an abundance of published data on their effects at the level of nociceptive behavior. The natural product agonists of TRPV3, carvacrol and eugenol, were reported to have antinociceptive effects in mice [58, 59] although it is unclear whether the effects are dependent on TRPV3. No data on pain behavior have been reported with incensole acetate, a relatively potent TRPV3 agonist. FPP, an endogenous TRPV3 agonist, was found to elicit thermal hyperalgesia when injected intradermally [32]. While FPP may be specific in the activation of TRPV3 among the TRPV family, it has also been described as a ligand for lysophosphatidic acid receptor 5 (or GPR92) [60]. Given the non-selective nature of many of the TRPV3 agonists, it may be useful to evaluate them in TRPV3 deficient animals or in conjunction with TRPV3 knockdown.

Several endogenous molecules such as isopentenyl pyrophosphate and resolvin D1 were reported to interact with TRPV3, as well as other thermo TRPs or receptors such as TLR4 [48, 51, 52, 61]. These molecules were reported to suppress inflammatory hyperalgesia induced by intradermal injection of complete Freund's adjuvant. ShRNA knockdown of TRPV3 blunted the effect of 17(R)-resolvin D1 [52]. The data suggest the likely existence of endogenous antagonists of TRPV3 that could contribute physiologically to nociception.

Multiple classes of small molecules described as selective antagonists of TRPV3 started appearing in the patent literature since 2006 from companies such as Hydra Biosciences and Glenmark Pharmaceuticals. These molecules were reported to be effective in suppressing inflammatory and neuropathic pain in animal models. Excellent reviews covering this topic have been published to which interested readers are referred $[62,63]$. If these compounds are indeed selective and potent at TRPV3, they could be extremely valuable in advancing our understanding of the biology of TRPV3.

\section{Altered Expression of TRPV3 Under Pathological Conditions in Rodents and Humans}

Changes in TRPV3 protein or mRNA expression have been investigated in humans and rodents under injury and pain states. No changes in TRPV3 mRNA levels in L4-L6 DRGs were observed in rats that had undergone the chronic constriction injury, on Day 7 or Day 14 post surgery when increased pain sensitivity was evident [64]. Another study also found no differences in TRPV3 mRNA levels in the L4 DRG at 4 days, 15 days or 3 months following the rat spared nerve injury [65]. In contrast, increased TRPV3 mRNA levels were described at 28 and 50 days after spinal nerve ligation in one report in the patent literature [66]. Although the gene expression studies do not provide causative evidence for a role of TRPV3 in pain states, they could be informative on the regulation of TRPV3. In addition to mRNA, analysis at the protein level using specific antibodies verified in knockout tissues could also shed light on this issue.

In humans, increased TRPV3 immunostaining was found in injured DRGs and peripheral nerves $[1,6]$. Higher levels of TRPV3 immunoreactivity were reported in keratinocytes of painful breast tissue, with the intensity of staining correlating to the intensity of pain [7]. In contrast, a trend for a decrease in TRPV3 immunoreactivity in keratinocytes was found in diabetic skin [6]. These changes in TRPV3 levels in humans suggest TRPV3 protein may be regulated in the context of injury or pain.

At the genetic level, a single nucleotide polymorphism in the TRPV3 gene was found to be associated with migraine with aura in a study of a population of Spanish Caucasian [67]. It would be interesting to conduct a larger scale analysis encompassing of case controlled population with different pain syndromes.

\section{Non-Sensory Functions of TRPV3 in Skin}

In considering TRPV3 as a potential target for clinical development of novel analgesics, one cannot overlook other physiological roles that TRPV3 might assume. One area that has received much attention is the involvement of TRPV 3 in keratinocyte biology and dermatological diseases.

\section{Hair Growth}

One of the first clues that implicated a role for TRPV3 in hair growth came from the generation of TRPV3 deficient animals. Moqrich and colleagues observed that TRPV3 deficient animals exhibited brief periods of ventral hair abnormality [24]. In an independently generated line of TRPV3 deficient mice, Cheng and colleagues observed wavy body hair and curly whiskers [8]. These findings are well matched with the strong expression of TRPV3 in keratinocytes including those surrounding the hair follicles. Histological examination revealed the hair follicles of these mice had misaligned orientations. The similarity of this phenotype to EGFR receptor deficient mice led Cheng and colleagues to study the interaction between EGFR and TRPV3 [8].

TRPV3 stimulation by chemical agonists was found to suppress proliferation and induce apoptosis, leading to inhibition of hair growth in primary cultures of human outer root sheath keratinocytes [14]. These proliferative and apoptotic effects could be rescued by TRPV3 knockdown. A mutation of a single amino acid residue of TRPV3 has been attributed to the hairless phenotype in the Ds-Nh mice a naturally occurring mutant line of mice [13]. The G573S mutation of TRPV 3 rendered TRPV 3 constitutively active [68], and transgenic mice overexpressing TRPV3 G573S has spontaneous hairlessness [69]. It is intriguing that both TRPV3 deficiency and overactivity resulted in abnormal hair phenotypes.

\section{Skin Architecture and Barrier Function}

Stimulation with TRPV3 agonists did not seem to affect the barrier recovery rate in wildtype human epidermal keratinocytes, or affect tight junctions and adherens junctions [70]. Investigation of TRPV3 knock out mice by 
Moqrich et al. [24] showed that there were no gross anatomical skin defects. Epidermal and dermal layer thickness, keratinocyte specific markers and skin epidermal barrier integrity were found to be normal. Other groups also reported no differences in barrier function based on biotin permeation in newborn and transepidermal water loss in the adult animals using the same.TRPV3-deficient mice on the C57/B16 background [71, 72].

However, another group reported discrepant results using independently generated lines of TRPV3-deficient mice [8]. Cheng and colleagues [8] reported that the $\mathrm{K} 1 / \mathrm{K} 10$ and loricrin positive layers were thicker, while the K14 positive layer was thinner compared to wildtype mice in neonatal TRPV3 deficient mice. These thickness changes indicated alterations in the proliferation/maturation rates of keratinocytes in their life cycle. Smaller/fragmented corneocytes (matured keratinocytes) were also observed. The skin architecture alterations could be independent of, or contribute to, the skin barrier defect seen in the E17 animals (measured by Toluidine Blue permeation). Measurable changes in the activity of transglutaminase, a cross-linking enzyme necessary for barrier function, likely underlie the functional defect. Hence it is clear that TRPV 3 contributes to the appropriate formation of skin epidermis and barrier during development. It is less clear, however, if TRPV3 plays an equally important role postnatally in these respects. The seemingly discrepant findings suggest that the barrier consequence of TRPV3 deficiency may depend on age and genetic background. Since multiple components in sum constitute the skin architecture and barrier, a comprehensive analysis of multiple components of skin architecture and barrier function may be necessary to determine the extent of TRPV3 involvement.

\section{Skin Inflammation}

Aside from hairlessness, TRPV3 G573S mutation was also found to be associated with the development of skin inflammation or dermatitis in multiple strains of mice [73]. The exact nature and extent of the inflammatory phenotype appears to vary depending on the genetic background. In the Ds background, G573S mutation caused spontaneous dermatitis but not hapten-induced dermatitis, whereas in the $\mathrm{NC} / \mathrm{Nga}$ background, hapten-induced dermatitis developed but not spontaneous dermatitis [74]. Increased scratching behavior and increased number of immune cells in the skin also accompanied the mutation. It is not entirely clear whether the itching is independent of dermatitis, although a recent study showing TRPV3 deficient mice to be resistant to the development of dry skin induced itch seems to indicate such possibility [75].

\section{TRPV3 and Human Skin Disorders}

Over the past year, there has been an explosion of human data on TRPV3 in various dermatological diseases. TRPV3 mRNA was found to be upregulated in both the lesioned and xerotic skin areas of atopic dermatitis patients, along with many genes related to inflammation [76]. Dermal TRPV3 and TRPV2 immunostaining was reported to be increased in erythematotelangiectatic rosacea, a chronic inflammatory skin disease [77]. Mutations of TRPV3 gene were found to be associated with Olmsted Syndrome, a rare congenital skin disorder characterized by periorificial keratoderma, alopecia and itching [78]. Intriguingly, 5 out of the 6 patients sequenced had mutation at the Gly573 residue, the same site as the naturally occurring mutant mouse line. These reports highlight the importance for proper TRPV3 function in skin physiology.

\section{Perspective: Targeting TRPV3 to develop Novel Analgesics}

Although there is not an overwhelming amount of evidence pointing to TRPV3 as a target for developing novel anti-hyperalgesic therapeutics as for other TRPs such as TRPV1 and TRPA1, some data supporting targeting TRPV3 for pain exists. Data from the genetically modified animals suggest TRPV3 contributes minimally to acute innocuous and noxious thermal sensation under normal physiology, in a gender and genetic background specific manner. Results from in vivo experiments with non-selective TRPV3 agonists and antagonists such as FPP and resolvins support the notion that modulation of TRPV3 activity could potentially have an impact on hyperalgesia. Information from the patent literature indicates promise for the utility of selective TRPV 3 antagonists in treating chronic pain of inflammatory or neuropathic nature. Moreover, GRC 15300, a TRPV3 antagonist from Glenmark Pharmaceuticals SA, (Inlicensed by Sanofi Avantis) recently completed Phase-I trials and a phase II clinical trial is expected to be underway in 2012 [63].

Despite interest in TRPV3 antagonists as novel therapeutics, some questions remain regarding whether TRPV3 constitutes a good target for therapy. First, convincing evidence for the contribution of TRPV3 to either development or maintenance of chronic pain have not been published in the peer-reviewed literature. Although preliminary reports on the effect of TRPV3 antagonists in chronic pain models exist $[62,79,80]$, further investigation using TRPV3 selective agonists, TRPV3 knockdown, or in the context of TRPV3 deficiency is warranted. Second, the potentially dissimilar expression profile of TRPV3 between humans and rodents might complicate preclinical/clinical translation into humans - accurate prediction of effective therapeutic doses may not be straightforward. Third, the substantial body of evidence linking TRPV3 to skin barrier function, hair growth, inflammation and dermatological diseases raises the question whether manipulation of TRPV3 activity might have an impact on skin biology. Results from gain of function studies demonstrated that TRPV3 overactivity can result in increased skin inflammation and skin diseases including Olmsted syndrome, suggesting TRPV3 antagonists could be beneficial. However, one line of TRPV3 deficient mice was found to be defective in the normal development of skin architecture and barrier function, pointing to a potential liability for TRPV3 antagonists. Interestingly, the mutants with overactive TRPV3 were hairless, while TRPV3 deficient animals developed wavy hair coat. The findings seem to point to the notion that a fine balance of TRPV3 activity is necessary for skin biology, and caution should be exercised in manipulating it. Furthermore, it is possible that straightforward targeting of TRPV3 using specific antagonists or agonists may not guarantee specific antihyperalgesic effects. Thus, it will be important to study the mechanisms involved in pathology- or injury-specific modulation of TRPV3. Targeting such pathology-dependent 
modulation of TRPV3 might minimize side effects related to non-sensory functions of TRPV3.

With the clinical development of TRPV3 antagonists, potentially exciting learning opportunities are on the horizon. More research into the effects and the mechanisms of selective modulation of TRPV3 will increase our understanding of the physiological and pathophysiological roles of TRPV3, and inform us in the design of effective therapies for pain and/or skin disorders.

\section{CONFLICT OF INTEREST}

S.M.H. is an employee of Abbott Laboratories during the preparation of this article.

\section{ACKNOWLEDGEMENTS}

This project is supported by NIH-NIDCR DE019694 and DE020866 to MKC.

\section{REFERENCES}

[1] Smith GD, Gunthorpe MJ, Kelsell RE, et al. TRPV3 is a temperature-sensitive vanilloid receptor-like protein. Nature 2002; 418(6894): 186-90.

[2] $\mathrm{Xu} \mathrm{H}$, Ramsey IS, Kotecha SA, et al. TRPV3 is a calciumpermeable temperature-sensitive cation channel. Nature 2002; 418(6894): 181-6.

[3] Peier AM, Reeve AJ, Andersson DA, et al. A heat-sensitive TRP channel expressed in keratinocytes. Science 2002; 296(5575): 2046-9.

[4] Caterina MJ, Schumacher MA, Tominaga M, Rosen TA, Levine JD, Julius D. The capsaicin receptor: a heat-activated ion channel in the pain pathway. Nature 1997; 389(6653): 816-24.

[5] Caterina MJ, Rosen TA, Tominaga M, Brake AJ, Julius D. A capsaicin-receptor homologue with a high threshold for noxious heat. Nature 1999; 398(6726): 436-41.

[6] Facer P, Casula MA, Smith GD, et al. Differential expression of the capsaicin receptor TRPV1 and related novel receptors TRPV3, TRPV4 and TRPM8 in normal human tissues and changes in traumatic and diabetic neuropathy. BMC Neurol 2007; 7: 11.

[7] Gopinath P, Wan E, Holdcroft A, et al. Increased capsaicin receptor TRPV1 in skin nerve fibres and related vanilloid receptors TRPV3 and TRPV4 in keratinocytes in human breast pain. BMC Womens Health 2005; 5(1): 2.

[8] Cheng X, Jin J, Hu L, et al. TRP channel regulates EGFR signaling in hair morphogenesis and skin barrier formation. Cell 2010; 141(2): 331-43.

[9] Ueda T, Yamada T, Ugawa S, Ishida Y, Shimada S. TRPV3, a thermosensitive channel is expressed in mouse distal colon epithelium. Biochem Biophys Res Commun 2009; 383(1): 130-4.

[10] Chung MK, Lee H, Mizuno A, Suzuki M, Caterina MJ. TRPV3 and TRPV4 mediate warmth-evoked currents in primary mouse keratinocytes. J Biol Chem 2004; 279(20): 21569-75.

[11] Chung MK, Lee H, Caterina MJ. Warm temperatures activate TRPV4 in mouse 308 keratinocytes. J Biol Chem 2003; 278(34): 32037-46.

[12] Huang SM, Lee H, Chung MK, et al. Overexpressed transient receptor potential vanilloid 3 ion channels in skin keratinocytes modulate pain sensitivity via prostaglandin E2. J Neurosci 2008; 28(51): 13727-37.

[13] Asakawa M, Yoshioka T, Matsutani T, et al. Association of a mutation in TRPV3 with defective hair growth in rodents. J Invest Dermatol 2006; 126(12): 2664-72.

[14] Borbiro I, Lisztes E, Toth BI, et al. Activation of transient receptor potential vanilloid-3 inhibits human hair growth. J Invest Dermatol 2011; 131(8): 1605-14.

[15] Xu H, Delling M, Jun JC, Clapham DE. Oregano, thyme and clovederived flavors and skin sensitizers activate specific TRP channels. Nat Neurosci 2006; 9(5): 628-35.

[16] Guler AD, Lee H, Iida T, Shimizu I, Tominaga M, Caterina M. Heat-evoked activation of the ion channel, TRPV4. J Neurosci 2002; 22(15): 6408-14.
[17] Chung MK, Guler AD, Caterina MJ. Biphasic currents evoked by chemical or thermal activation of the heat-gated ion channel, TRPV3. J Biol Chem 2005; 280(16): 15928-41.

[18] Doerner JF, Hatt H, Ramsey IS. Voltage- and temperaturedependent activation of TRPV3 channels is potentiated by receptormediated PI(4,5)P2 hydrolysis. J Gen Physiol 2011; 137(3): 27188 .

[19] Grandl J, Hu H, Bandell M, et al. Pore region of TRPV3 ion channel is specifically required for heat activation. Nat Neurosci 2008; 11(9): 1007-13.

[20] Xiao R, Tang J, Wang C, Colton CK, Tian J, Zhu MX. Calcium plays a central role in the sensitization of TRPV3 channel to repetitive stimulations. J Biol Chem 2008; 283(10): 6162-74.

[21] Yao J, Liu B, Qin F. Modular thermal sensors in temperature-gated transient receptor potential (TRP) channels. Proc Natl Acad Sci USA $2011 ; 108(27): 11109-14$.

[22] $\mathrm{Hu} \mathrm{HZ,} \mathrm{Gu} \mathrm{Q,} \mathrm{Wang} \mathrm{C,} \mathrm{et} \mathrm{al.} \mathrm{2-aminoethoxydiphenyl} \mathrm{borate} \mathrm{is} \mathrm{a}$ common activator of TRPV1, TRPV2, and TRPV3. J Biol Chem 2004; 279(34): 35741-8.

[23] Chung MK, Lee H, Mizuno A, Suzuki M, Caterina MJ. 2aminoethoxydiphenyl borate activates and sensitizes the heat-gated ion channel TRPV3. J Neurosci 2004; 24(22): 5177-82.

[24] Moqrich A, Hwang SW, Earley TJ, et al. Impaired thermosensation in mice lacking TRPV3, a heat and camphor sensor in the skin. Science 2005; 307(5714): 1468-72.

[25] Macpherson LJ, Hwang SW, Miyamoto T, Dubin AE, Patapoutian A, Story GM. More than cool: promiscuous relationships of menthol and other sensory compounds. Mol Cell Neurosci 2006; 32(4): 335-43.

[26] Vogt-Eisele AK, Weber K, Sherkheli MA, et al. Monoterpenoid agonists of TRPV3. Br J Pharmacol 2007; 151(4): 530-40.

[27] Yang BH, Piao ZG, Kim YB, et al. Activation of vanilloid receptor 1 (VR1) by eugenol. J Dent Res 2003; 82(10): 781-5.

[28] Xu H, Blair NT, Clapham DE. Camphor activates and strongly desensitizes the transient receptor potential vanilloid subtype 1 channel in a vanilloid-independent mechanism. J Neurosci 2005; 25(39): 8924-37.

[29] Stotz SC, Vriens J, Martyn D, Clardy J, Clapham DE. Citral sensing by Transient [corrected] receptor potential channels in dorsal root ganglion neurons. PLoS ONE 2008; 3(5): e2082.

[30] Moussaieff A, Rimmerman N, Bregman T, et al. Incensole acetate, an incense component, elicits psychoactivity by activating TRPV3 channels in the brain. FASEB J 2008; 22(8): 3024-34.

[31] De Petrocellis L, Orlando P, Moriello AS, et al. Cannabinoid actions at TRPV channels: effects on TRPV3 and TRPV4 and their potential relevance to gastrointestinal inflammation. Acta Physiol (Oxf) 2012; 204(2): 255-66.

[32] Bang S, Yoo S, Yang TJ, Cho H, Hwang SW. Farnesyl pyrophosphate is a novel pain-producing molecule via specific activation of TRPV3. J Biol Chem 2010; 285(25): 19362-71.

[33] $\mathrm{Hu} \mathrm{H}$, Grandl J, Bandell M, Petrus M, Patapoutian A. Two amino acid residues determine 2-APB sensitivity of the ion channels TRPV3 and TRPV4. Proc Natl Acad Sci USA 2009; 106(5): 162631.

[34] Chung MK, Jung SJ, Oh SB. Role of TRP channels in pain sensation. Adv Exp Med Biol 2011; 704: 615-36.

[35] Prescott ED, Julius D. A modular PIP2 binding site as a determinant of capsaicin receptor sensitivity. Science 2003; 300(5623): 1284-8.

[36] $\mathrm{Hu} \mathrm{HZ,} \mathrm{Xiao} \mathrm{R,} \mathrm{Wang} \mathrm{C,} \mathrm{et} \mathrm{al.} \mathrm{Potentiation} \mathrm{of} \mathrm{TRPV3} \mathrm{channel}$ function by unsaturated fatty acids. J Cell Physiol 2006; 208(1): 201-12.

[37] Hwang SW, Cho H, Kwak J, et al. Direct activation of capsaicin receptors by products of lipoxygenases: endogenous capsaicin-like substances. Proc Natl Acad Sci USA 2000; 97(11): 6155-60.

[38] Tominaga M, Caterina MJ, Malmberg AB, et al. The cloned capsaicin receptor integrates multiple pain-producing stimuli. Neuron 1998; 21(3): 531-43.

[39] Phelps CB, Wang RR, Choo SS, Gaudet R. Differential regulation of TRPV1, TRPV3, and TRPV4 sensitivity through a conserved binding site on the ankyrin repeat domain. J Biol Chem 2010; 285(1): 731-40.

[40] Lishko PV, Procko E, Jin X, Phelps CB, Gaudet R. The ankyrin repeats of TRPV1 bind multiple ligands and modulate channel sensitivity. Neuron 2007; 54(6): 905-18. 
[41] Liu B, Yao J, Zhu MX, Qin F. Hysteresis of gating underlines sensitization of TRPV3 channels. J Gen Physiol 2011; 138(5): 50920.

[42] Chung MK, Guler AD, Caterina MJ. TRPV1 shows dynamic ionic selectivity during agonist stimulation. Nat Neurosci 2008; 11(5): 555-64.

[43] Karashima Y, Prenen J, Talavera K, Janssens A, Voets T, Nilius B. Agonist-Induced changes in $\mathrm{Ca} 2+$ Permeation through the nociceptor cation channel TRPA1. Biophys J 2010; 98(5): 773-83.

[44] Chandrashekar J, Hoon MA, Ryba NJ, Zuker CS. The receptors and cells for mammalian taste. Nature 2006; 444(7117): 288-94.

[45] Fuchs PA, Glowatzki E, Moser T. The afferent synapse of cochlear hair cells. Curr Opin Neurobiol 2003; 13(4): 452-8.

[46] Burnstock G. Purinergic mechanosensory transduction and visceral pain. Mol Pain 2009; 5: 69.

[47] Miyamoto T, Petrus MJ, Dubin AE, Patapoutian A. TRPV3 regulates nitric oxide synthase-independent nitric oxide synthesis in the skin. Nat Commun 2011; 2: 369.

[48] Bang S, Yoo S, Yang TJ, Cho H, Hwang SW. Isopentenyl pyrophosphate is a novel antinociceptive substance that inhibits TRPV3 and TRPA1 ion channels. Pain 2011; 152(5): 1156-64.

[49] Xu ZZ, Zhang L, Liu T, et al. Resolvins RvE1 and RvD1 attenuate inflammatory pain via central and peripheral actions. Nat Med 2010; 16(5): 592-7.

[50] Lima-Garcia JF, Dutra RC, da Silva K, Motta EM, Campos MM, Calixto JB. The precursor of resolvin D series and aspirin-triggered resolvin D1 display anti-hyperalgesic properties in adjuvantinduced arthritis in rats. Br J Pharmacol 2011; 164(2): 278-93.

[51] Bang S, Yoo S, Yang TJ, Cho H, Kim YG, Hwang SW. Resolvin D1 attenuates activation of sensory transient receptor potential channels leading to multiple anti-nociception. $\mathrm{Br} \mathrm{J}$ Pharmacol 2010; 161(3): 707-20

[52] Bang S, Yoo S, Yang TJ, Cho H, Hwang SW. 17(R)-resolvin D1 specifically inhibits transient receptor potential ion channel vanilloid 3 leading to peripheral antinociception. Br J Pharmacol 2012; 165(3): 683-92.

[53] Caterina MJ, Leffler A, Malmberg AB, et al. Impaired nociception and pain sensation in mice lacking the capsaicin receptor. Science 2000; 288(5464): 306-13.

[54] Lee H, Caterina MJ. TRPV channels as thermosensory receptors in epithelial cells. Pflugers Arch 2005; 451(1): 160-7.

[55] Lumpkin EA, Caterina MJ. Mechanisms of sensory transduction in the skin. Nature 2007; 445(7130): 858-65.

[56] Mandadi S, Sokabe T, Shibasaki K, et al. TRPV3 in keratinocytes transmits temperature information to sensory neurons via ATP. Pflugers Arch 2009; 458(6): 1093-102.

[57] Huang SM, Li X, Yu Y, Wang J, Caterina MJ. TRPV3 and TRPV4 ion channels are not major contributors to mouse heat sensation. Mol Pain 2011; 7: 37.

[58] Guimaraes AG, Oliveira GF, Melo MS, et al. Bioassay-guided evaluation of antioxidant and antinociceptive activities of carvacrol. Basic Clin Pharmacol Toxicol 2010; 107(6): 949-57.

[59] Park SH, Sim YB, Lee JK, et al. The analgesic effects and mechanisms of orally administered eugenol. Arch Pharm Res 2011; 34(3): 501-7.

[60] Oh DY, Yoon JM, Moon MJ, et al. Identification of farnesyl pyrophosphate and $\mathrm{N}$-arachidonylglycine as endogenous ligands for GPR92. J Biol Chem 2008; 283(30): 21054-64.

[61] Palmer CD, Mancuso CJ, Weiss JP, Serhan CN, Guinan EC, Levy O. 17(R)-Resolvin D1 differentially regulates TLR4-mediated responses of primary human macrophages to purified LPS and live E. coli. J Leukoc Biol 2011; 90(3): 459-70.
[62] Khairatkar-Joshi N, Maharaj N, Thomas A. The TRPV3 receptor as a pain target: A therapeutic promise or just some more new biology?. Open Drug Discov J 2010; 2(1): 89-97.

[63] Reilly RM, Kym PR. Analgesic potential of TRPV3 antagonists. Curr Top Med Chem 2011; 11(17): 2210-5.

[64] Frederick J, Buck ME, Matson DJ, Cortright DN. Increased TRPA1, TRPM8, and TRPV2 expression in dorsal root ganglia by nerve injury. Biochem Biophys Res Commun 2007; 358(4): 105864.

[65] Staaf S, Oerther S, Lucas G, Mattsson JP, Ernfors P. Differential regulation of TRP channels in a rat model of neuropathic pain. Pain 2009; 144(1-2): 187-99.

[66] Bevan S, Ganju P, McIntyre P, Patapoutian A, Peier AM, Song C, Inventors. Transient receptor potential 3 and its use. US patent 7638601-B2/63, 2009.

[67] Carreno O, Corominas R, Fernandez-Morales J, et al. SNP variants within the vanilloid TRPV1 and TRPV3 receptor genes are associated with migraine in the Spanish population. Am J Med Genet B Neuropsychiatr Genet 2012; 159B(1): 94-103.

[68] Xiao R, Tian J, Tang J, Zhu MX. The TRPV3 mutation associated with the hairless phenotype in rodents is constitutively active. Cell Calcium 2008; 43(4): 334-43.

[69] Imura K, Yoshioka T, Hikita I, et al. Influence of TRPV3 mutation on hair growth cycle in mice. Biochem Biophys Res Commun 2007; 363(3): 479-83.

[70] Kida N, Sokabe T, Kashio M, et al. Importance of transient receptor potential vanilloid 4 (TRPV4) in epidermal barrier function in human skin keratinocytes. Pflugers Arch 2012; 463(5): 715-25.

[71] Denda M, Sokabe T, Fukumi-Tominaga T, Tominaga M. Effects of skin surface temperature on epidermal permeability barrier homeostasis. J Invest Dermatol 2007; 127(3): 654-9.

[72] Sokabe T, Fukumi-Tominaga T, Yonemura S, Mizuno A, Tominaga M. The TRPV4 channel contributes to intercellular junction formation in keratinocytes. J Biol Chem 2010; 285(24): $18749-58$.

[73] Yoshioka T, Imura K, Asakawa M, et al. Impact of the Gly573Ser substitution in TRPV3 on the development of allergic and pruritic dermatitis in mice. J Invest Dermatol 2009; 129(3): 714-22.

[74] Imura K, Yoshioka T, Hirasawa T, Sakata T. Role of TRPV3 in immune response to development of dermatitis. J Inflamm (Lond) 2009; 6: 17.

[75] Yamamoto-Kasai E, Imura K, Yasui $\mathrm{K}$, et al. TRPV3 as a therapeutic target for itch. J Invest Dermatol 2012; 132(8): 210912.

[76] Bianchi P, Ribet V, Casas C, Lejeune O, Schmitt AM, Redoules D. Analysis of gene expression in atopic dermatitis using a microabrasive method. J Invest Dermatol 2012; 132(2): 469-72.

[77] Sulk M, Seeliger S, Aubert J, et al. Distribution and expression of non-neuronal transient receptor potential (TRPV) ion channels in rosacea. J Invest Dermatol 2012; 132(4): 1253-62.

[78] Lin Z, Chen Q, Lee M, et al. Exome sequencing reveals mutations in TRPV3 as a cause of Olmsted syndrome. Am J Hum Genet 2012; 90(3): 558-64.

[79] Gullapalli S. GRC 15300 - A novel, selective, orally active TRPV3 antagonist in clinical trial for potential treatment of chronic inflammatory and neuropathic pain. CHI's World Pharma Congress on Targeting pain with novel targets; 2010 June 15-17; Philadelphia, PA: USA 2010.

[80] Khairatkar-Joshi N. TRPV3 antagonists for treatment of pain Generation next? Pain Therapeutics meeting of the SMi; 2008 June 10; London: UK 2008.

\section{(C) Huang and Chung; Licensee Bentham Open.}

This is an open access article licensed under the terms of the Creative Commons Attribution Non-Commercial License (http://creativecommons.org/licenses/by-nc/3.0/) which permits unrestricted, non-commercial use, distribution and reproduction in any medium, provided the work is properly cited. 